\title{
Clinical genetics provider real-time workflow study
}

\author{
Elizabeth McPherson, $M D^{1}$, Christina Zaleski, $M S^{1}$, Katrina Benishek ${ }^{2}$, Catherine A. McCarty, MPH, PhD ${ }^{3}$,
} Philip F. Giampietro, $\mathrm{MD}, \mathrm{PhD}^{1}$, Kara Reynolds, $M S^{1}$, and Kristen Rasmussen, $M S^{1}$

\begin{abstract}
Purpose: Our work is the first documentation, in real time, of workflow in a general genetics department including data on patient care, research, and other activities for both clinical geneticists and genetic counselors. Methods: All physician geneticists and genetic counselors in the medical genetics department used an electronic tool to record their activities in 15 minute increments during clinic hours, evenings, and weekends over a 10-week period.

Results: The average work week was 54.1 hours for physicians and 43.5 hours for genetic counselors. During clinic hours physicians spent about one-fourth of their time on direct patient care, one-fourth on other patient-related activities, one-fourth on research unrelated to individual patient care, and the remaining fourth on all other activities. However, after hours and on weekends they spent most of their time on research. Genetic counselors spent half of their time on patient-related activities, one-fourth on direct patient care, and the remainder on all other activities. The total professional time averaged 7 hours per new patient and 3.5 hours per follow-up with nearly $60 \%$ of this time devoted to patient-related activities. Conclusions: The labor intensive nature of clinical genetics, the large amount of time devoted to patient-related activities, and continuing limitations on billing by genetic counselors all contribute to the financial challenges faced by genetics departments. Genet Med 2008:10(9):699-706.
\end{abstract}

Key Words: clinical genetics services, workforce, workflow, time study, reimbursement, genetic counseling

Provision of medical genetics services is a time and labor intensive activity. ${ }^{1,2}$ The detailed three generation pedigree not only takes far longer than the standard family history screening in primary care (which adds an average of only 3 min to a primary care visit ${ }^{3}$ ), but frequently must be supplemented by acquisition and review of medical records of affected relatives, examination of parents and other relatives (who don't usually have their own appointments), and the review of family photographs when affected relatives are unavailable. Because of the complexity of modern genetic knowledge and the need to discuss implications for multiple family members, face-to-face genetic counseling is time-consuming. The ordering, insurance preauthorization, and follow-up of increasingly complex laboratory tests, literature review for rare diagnoses, and documentation of the visit (which is more extensive than in most disciplines due to the need to educate referring providers about rare disorders), as well as writing and reviewing of detailed summary letters for the family also contribute to the work of genetic counseling. Although some of these challenges exist in other fields, genetics remains unique in terms of dependence

\footnotetext{
From the ${ }^{1}$ Department of Medical Genetic Services, Marshfield Clinic, Marshfield, Wisconsin ${ }^{2}$ Systems and Processes, Marshfield Clinic, Marshfield, Wisconsin; and ${ }^{3}$ Center for Human Genetics, Marshfield Clinic Research Foundation, Marshfield, Wisconsin.

Elizabeth McPherson, MD, Department of Medical Genetic Services 3C1, Marshfield Clinic, 1000 North Oak Avenue, Marshfield, WI 54449. E-mail: mcpherson.elizabeth@ marshfieldclinic.org.

Disclosure: The authors declare no conflict of interest.

Submitted for publication March 14, 2008.

Accepted for publication May 28, 2008.

DOI: 10.1097/GIM.0b013e318182206f
}

on family information. Furthermore, because most genetic disorders are rare, time savings through standardization of protocols, documentation tools, and patient education materials are rarely applicable. Rapid advances in genetic knowledge have resulted in exponential increases in the possible laboratory tests and diagnoses. With the development of personalized medicine, choice of genetic tests and interpretation of the results will become an increasingly important aspect of care for common disorders, placing an increasing demand on available genetics services.

Previous studies of workflow in clinical genetics have been very limited. The initial studies of time allocation in an adult genetics clinic 20 years ago documented that geneticists and genetic counselors, as well as a social worker, clinic coordinator, and secretary spent an average time of 7 hours per new patient (including $4 \mathrm{hr}$ in clinic and $3 \mathrm{hr}$ in preparation and follow-up) and 4 hours per returning patient (including $2 \mathrm{hr}$ in clinic and $2 \mathrm{hr}$ in preparation and follow-up). ${ }^{1}$ Preparation and follow-up activities included records review, literature search, telephone follow-up, and correspondence. The clinic was based in an academic institution and the greatest portion of the time was spent by fellows, but attending physician time averaged 90 minutes per new patient and 79 minutes per follow-up. Genetic counselors saw only selected patients, but when involved spent an average of 61 minutes per new patient and 28 minutes per follow-up. All the physicians and genetic counselors were involved in and partially supported by other activities, such as teaching and research.

Extending their study to additional settings, Bernhardt and Pyerit $\mathrm{z}^{4}$ evaluated the time devoted to new and follow-up pa- 
Table 1

Previous clinical genetics workflow studies

\begin{tabular}{|c|c|c|c|c|c|c|c|c|c|}
\hline \multirow[b]{2}{*}{ Study } & \multirow[b]{2}{*}{ Clinic type } & \multicolumn{4}{|c|}{ New (hr) } & \multicolumn{4}{|c|}{ Follow-up (hr) } \\
\hline & & $\begin{array}{c}\text { Total } \\
\text { face-to-face }\end{array}$ & $\begin{array}{c}\text { Total } \\
\text { patient-related } \\
\text { activity }^{a}\end{array}$ & $\mathrm{MD}^{b}$ & Non-MD ${ }^{b}$ & $\begin{array}{c}\text { Total } \\
\text { face-to-face }{ }^{a}\end{array}$ & $\begin{array}{c}\text { Total } \\
\text { patient-related } \\
\text { activity }^{a}\end{array}$ & $\mathrm{MD}^{b}$ & Non-MD ${ }^{b}$ \\
\hline Bernhardt $1987^{1}$ & Adult & 4 & 3 & $1.5^{c}$ & - & 2 & 2 & $1.3^{\mathrm{c}}$ & - \\
\hline \multirow[t]{4}{*}{ Bernhardt $1989^{4}$} & Private & 2.6 & 3.1 & 1.7 & 2.3 & 1.6 & 1.2 & 1.1 & $1 \mathrm{hr}$ \\
\hline & Pediatric & 1.5 & 2.6 & 1.9 & 0.6 & 1.3 & 2.6 & 2.1 & 0.3 \\
\hline & Outreach & 1.5 & 3.6 & 1.6 & 2.0 & 1.3 & 3.1 & 1.6 & 1.5 \\
\hline & Prenatal & 1.4 & 1.6 & 0.2 & 1.9 & - & - & - & - \\
\hline Pletcher $2002^{5}$ & All & - & - & 3.2 & - & - & - & - & - \\
\hline Cooksey $2005^{2}$ & All & - & - & 2.5 & - & - & - & 1.5 & - \\
\hline
\end{tabular}

${ }^{a}$ Includes MD and non-MD time.

${ }^{b}$ Included both face-to-face and patient related activity time.

${ }^{c}$ Attending MD only.

tients in four different clinical settings including a private genetics specialty clinic, a pediatric genetics clinic, a genetics outreach clinic, and a prenatal genetics clinic. Unlike the original study, none of these included time spent by fellows. The total times and face-to-face times varied somewhat by clinic type but were somewhat shorter than for the previous study (see Table 1). The total time requirements for a new patient ranged from 5.7 hours in the specialty clinic to only 3 hours in the prenatal clinic. Follow-up visits (excluding prenatal clinic where follow-ups were not routinely scheduled) ranged from 50 to $90 \%$ of the initial visit time. As in the previous study, at least half the total time was spent before and after the clinic visit. Physician time (including preparation, face-to-face, and follow-up) was 1.5 to 2 hours except in prenatal clinic where it was only 11 minutes. Non-MD professional time was approximately 2 hours per new patient except in pediatric genetics clinic. The slightly shorter total clinic times, longer time spent by non-MD professionals, and slightly longer time spent by attending physicians relative to the original study probably reflect the absence of fellows and greater involvement of genetic counselors compared with the original study.

Since that time, benefits from increasing efficiency and shifting of services from physicians to genetic counselors have been partially counter-balanced by increasing complexity of patients. In 2002, Pletcher et al. ${ }^{5}$ reported results of a survey in which clinical geneticists indicated that they spent an average of 3.1 hour of total time per new patient including not only direct face-to-face patient contact, but also preparation, dictation, and other follow-up. Time spent by genetic counselors was not included. A 2003 national survey of medical geneticists documented that those seeing a mixture of pediatric and adult patients spent a median of 75 minutes in direct patient care for each new genetics patient and 45 minutes per follow-up patient. ${ }^{2}$ The survey did not address other patient-related activities (PRA), such as records review, literature search, telephone contacts, and correspondence, but based on the weekly data given for numbers of new and follow-up patients and hours devoted to "clinical activity," it is possible to deduce that only about $50 \%$ of the "clinical" time was devoted to direct patient care. More information is needed on the time devoted to other usually nonreimbursable PRA.

With increasing pressure for cost effective medical care, more information is urgently needed regarding the time and effort required for provision of medical genetics services. Strengths of the 1987 study include the prospective design and the documentation for all PRAs, but the results may not reflect current practice. ${ }^{1}$ The $2002^{5}$ and $2003^{2}$ surveys were retrospective and thus subject to recall bias. Both surveys included all board certified clinical geneticists regardless of the proportion of time devoted to patient care. In the 2002 survey, all PRAs were combined with no attempt to determine the proportion of time spent face-to-face. PRA other than face-to-face clinic time was not specifically documented in the 2003 survey. Another area in which data are lacking is the role of the genetic counselor. The 1987 study ${ }^{1}$ reflected practice at the time in which genetic counselors saw only a minority of patients. Genetic counselors were not included in the 2002 or 2003 surveys. Among the clinical geneticists responding to the 2003 genetics workforce survey, $85 \%$ worked with one or more counselors and $30 \%$ noted an increase in their use of genetic counselors over the several years preceding the 2003 survey, ${ }^{2}$ but no recent survey of the time spent by genetic counselors in a general genetics clinic has been undertaken. More data are urgently needed regarding the workflow in clinical genetics, particularly the role of genetic counselors.

The Marshfield Clinic includes 40 centers throughout northern, central, and western Wisconsin. With more than 730 physicians in 80 medical specialties and subspecialties and a population of 350,000 unique patients receiving care, the Marshfield Clinic is one of the largest private, multispecialty group practices in the United States. In addition to providing clinical service, the clinic and geneticists within the clinic have a strong commitment to research and to genetic education for the provider and community. The Department of Medical Ge- 
netic Services supports two clinical geneticists (1.4 FTE combined clinical time, 0.5 FTE research, and 0.1 FTE administration) and three genetic counselors (2.3 FTE combined clinical time, 0.5 FTE for lab callbacks, and 0.2 FTE research) who offer comprehensive evaluation, counseling and co-ordination of care for children, and adults who are affected by or at risk for inherited disorders. Patient referrals include preconceptual, prenatal, pediatric, stillborn evaluations, adult oncology, neurology, and cardiology indications. Outreach is provided to three regional centers, averaging 2.5 days dedicated monthly. Telehealth consults are available from regional centers for oncology and preconceptual patients. Every patient referral includes face-to-face (or telehealth) time with both a genetic counselor and a medical geneticist. Clinics are held from 8:00 to $12: 00$ and 13:00 to 17:00 every weekday. From a departmental perspective this is considered "clinic time" although not all department personnel might be participating on a given day. Each physician is scheduled for 7 hours of patient appointments and 1 hour of PRA time per clinical day or for 8 hours of research time per research day according to his or her FTE's. New pediatric and adult patients are scheduled for 2 hours while follow-up and prenatal patients are allocated 1 hour each. Because the length of the appointments was set to accommodate the time needed by both physician and genetic counselor, the physician may be able to work on other activities while the genetic counselor is obtaining the history. Therefore, a genetic counselor sees fewer patients weekly than a physician. Double booking of physicians is allowed for patients seen primarily by the genetic counselor such as prenatal patients. Because of the complexity of scheduling, the need to save some slots for urgent prenatal visits and inpatient consults, and the significant number of no-shows and short-term cancellations, only about $80 \%$ of possible patient slots are actually filled.

In conjunction with a Marshfield Clinic initiative to improve patient access, the Department of Medical Genetic Services decided to undertake a workforce study with documentation of all time spent by genetics providers over a 10-week period. The primary goal for the study was to document the current workload, particularly the time spent in face-to-face patient contact and other PRA versus that devoted to nonpatient care activities such as research, administration, and teaching. A secondary goal was to understand the implications of workflow and scheduling limitations on projections of future growth opportunities and resource needs.

\section{MATERIALS AND METHODS}

The study goals required consistent documentation of activity as it occurred rather than retrospectively. An electronic collection tool was deemed most practical as all genetic counselors and geneticists in the department have convertible laptops. A Microsoft Excel spreadsheet was developed so each provider could log his or her time and activity. The spreadsheet was formatted so every 15 minutes between the hours of 7:00 and 22:00 had a cell dedicated for activity. The group defined task categories to maintain consistency of documentation. Clinical task categories included patient appointment/consult (direct face-to-face time actually spent with the patient), PRA (all non-face-to-face time devoted to patient care). PRA encompassed all activities on behalf of the patient such as records review, literature search, dictation and proofreading of letters, discussion of the case with other providers, insurance appeals, and telephone follow-up. Research (even if patients were involved as subjects) and teaching (even if the patient was used as an example) were not included in PRA unless they were intended to benefit a specific patient. Other nonclinical tasks included administrative, education (continuing medical education [CME], reading journals not related to a specific patient or research project, etc.), institutional review board (one MD and one genetic counselor are on the local institutional review board), laboratory reporting (primarily abnormal quad screen and cystic fibrosis carrier tests for the institution), and other (which turned out to be almost exclusively business travel). Personal time taken during the workday (lunch, etc.) was also recorded. The collection tool was tested for several days by the entire team to ensure that it could be used during the workday. If several activities occurred during a 15-minute slot, only the primary one was documented. Providers kept the activity log for 10 full weeks between April 28, 2007 and July 8, 2007. The logs for the two groups, genetic counselors and geneticists, were compiled and transferred into SAS JMP software (SAS Institute, Cary, NC) for analysis. Time slots left undocumented because of vacation or other absences were removed from the data. The time spent on each task was analyzed by provider type (genetic counselors and geneticists). Since genetics, like all departments in the Marshfield Clinic, is required to be open and providing clinical services 8:00 to 17:00, Monday through Friday, we separately analyzed time spent during "clinic hours" (8:00 to 17:00, Monday through Friday), "weekday pre- and postclinic hours" (pre-8:00 and post-17:00, Monday through Friday), and "weekends" (all time documented during Saturdays and Sundays).

\section{RESULTS}

Compliance with the recording procedures was excellent. After correction for holidays and scheduled vacation, there were 1971 possible "clinic hours" during the recording period of which usable data were recorded for 1807 (92\%). The missing data were attributable mainly to computer malfunctions and there is no reason to believe it would have been substantially different from that collected. Although there is no comparable method to evaluate the completeness of the evening and weekend data, it is assumed to be similar.

The three genetic counselors logged a total of 1099 hours. Corrected for vacations, holidays, and missing data, this gives an average of 43.5 hours per week (exclusive of time logged as personal). The two physicians logged a total of 948 hours. With similar corrections, this gives an average of 54.1 hour of work per week for physicians. Employees are expected to be in the clinic from 8:00 to 17:00 on weekdays with the assumption that, allowing 1 hour for lunch, they will work at least a 40- 
hour week. Meetings or other work during lunch are not unusual and on the average the genetic counselors worked 41.4 hours per week and the physicians worked 42.7 hours per week during the hours the clinic was open. The remaining time by the counselors was mostly spent during pre- and postclinic hours on weekdays that averaged 1.7 hours per week. Counselors only very occasionally logged weekend time that averaged $<0.5$ hours per weekend. Physicians worked an average of 7.8 hours per week during pre- and postclinic hours and 3.5 hours per weekend. The distribution and temporal pattern of activities differed by provider type and were tabulated separately for genetic counselors and physicians.

Overall, the genetic counselors spent almost half of their time (47.9\%) on PRAs followed by direct patient care (25.9\%), administrative activities (13.0\%), research (6.7\%), and $<5 \%$ each on business travel, teaching, laboratory reporting, education/CME, and institutional review board (Fig. 1). This pattern was essentially the same during "clinic hours" when genetic counselors spent $48.0 \%$ of their time on PRA, $27.0 \%$ on direct patient care, $12.2 \%$ on administrative activities, and $6.9 \%$ hours on research. During weekday pre- and postclinic hours the genetic counselors spent $36.6 \%$ of their time on PRA, $34.2 \%$ on administrative activities, $19.2 \%$ on business travel, and $<5 \%$ each on patient care, research, and teaching.

Physicians logged a total of 948 hours (excluding personal time). They spent the largest part of this time $29.4 \%$ on research followed by $24.4 \%$ on PRA, $18.3 \%$ on direct patient care, $11.9 \%$ on business travel, $6.8 \%$ on administrative activities, $5.4 \%$ on teaching, and the remainder on education/CME and institutional review board (Fig. 2). During clinic hours physicians' time was almost evenly divided among PRAs $(27.6 \%)$, research $(24.3 \%)$, and direct patient care $(23.2 \%)$ with the remaining time devoted to business travel (10.6\%), administrative (6.9\%), and teaching (3.7\%). Pre- and postclinic hours the physicians spent $40.5 \%$ of their time on research, with the remainder including business travel (18.6\%),

Weekday $8-5$
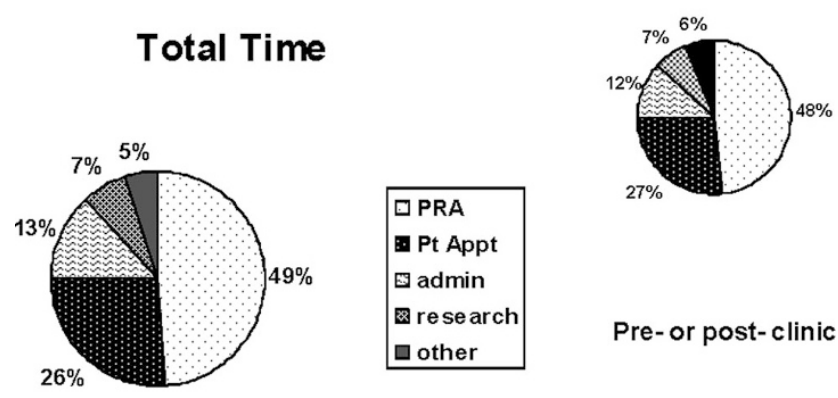

Pre-or post-clinic

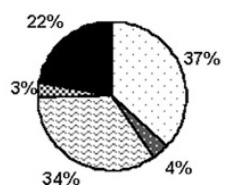

Fig. 1. Task distribution for genetic counselors.
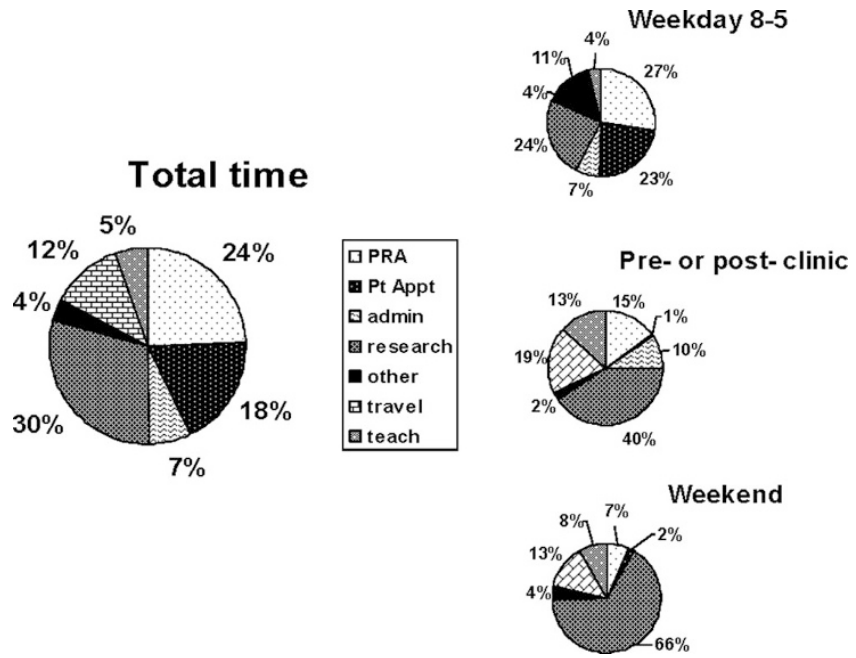

Fig. 2. Task distribution for physicians.

PRA (15.0\%), teaching (13\%), and administration (9.5\%). On weekends, physicians spent most of their time $(66.4 \%)$ on research plus $6 \%$ on business travel, $8.4 \%$ on teaching, and $6.7 \%$ on PRA.

During the recording period, the department saw 234 patients including 89 new patients or inpatient consults (usually assigned to $2 \mathrm{hr}$ time slots) as well as 86 follow-up patients and 58 prenatal patients usually assigned to 1 hour time slots (Table 2). Almost half of the new patients had known diagnoses including 29 cancer patients (breast, ovarian, colon, and other), 5 families seeking presymptomatic diagnosis of Huntington or other neurodegenerative disorders, 3 for preconceptual issues, and 6 with other diagnoses known at the time of referral (4 chromosomal, 2 neurofibromatosis). These patients with known diagnoses typically required less physician time than the remainder, but the genetic counselor time was longer due to the detailed scientific and psychosocial counseling provided, efforts to help families with insurance issues, and the extensive written and telephone follow-up. The undiagnosed patients included 24 with multiple anomalies/mental retardation, 3 stillbirths, and 20 with other incompletely diagnosed disorders (connective tissue disorders such as Ehlers Danlos, cardiac disorders, neuromuscular disorders, suspected skeletal dysplasias, metabolic disorders, etc). Three individuals referred for family history of specific rare syndromes were not affected with the familial condition but were found to have unrelated anomalies. Definitive diagnoses were made or suspected diagnoses were ruled out in about half of the patients with initially uncertain diagnosis. Two thirds of the prenatal cases had straightforward indications such as advanced maternal age, or abnormal maternal serum screen, although some of these had complex social issues or severe anxiety requiring additional counseling. One third of the prenatal cases had complex indications including serious fetal anomalies or combined maternal and fetal health problems. These cases required very extensive support during their decision-making process followed by either development of a birth plan or referrals for termination 
Table 2

Breakdown of patient types

\begin{tabular}{lr}
\hline Patient type & Count \\
\hline New visits & 24 \\
MCA/MR & 23 \\
Other unknown/incomplete diagnosis & 29 \\
Cancer & 5 \\
Huntington & 3 \\
Preconceptual & 6 \\
Other known & 16 \\
Prenatal & 16 \\
AMA & 6 \\
Abnormal maternal serum screen & 20 \\
Other routine & \\
Complex & 28 \\
Follow-up & 36 \\
New or changed diagnosis & 22 \\
Known diagnosis & \\
Unknown diagnosis & ad- \\
\hline MCA/MR, multiple congenital anomalies/mental retardation; & AMA \\
vanced maternal age. & \\
\hline
\end{tabular}

(which is not available locally). Among the follow-up patients about one-third were returning for discussion of new or changed diagnoses, just over one-third had known diagnoses requiring co-ordination of care and nearly one-third were followed for unknown diagnosis. Follow-up was by telephone only for most cancer patients (32\% of total new patients) and normal prenatal results ( $66 \%$ of all prenatals). Most other laboratory results were initially reported by telephone but all of these patients were also offered follow-up (immediate in the case of an abnormal test result or long term for undiagnosed patients).

Attempts to distinguish current from past or future patients were not entirely successful due to batching of tasks such as proof-reading of letters that might involve both, but it seemed that more than $95 \%$ of the PRA time was devoted to active patients, i.e., those seen within the 10 week period. Since the design of the study did not permit assignment of time spent to specific patients or even to classes of patients, the data were analyzed according to patient slots (with 2 for each new patient and 1 for each follow-up or prenatal) giving a total of 323 1-hour patient slots. Each patient was seen by both a genetic counselor and a physician. During each 1-hour patient slot the average time spent in direct patient care was 0.8 hours by the genetic counselor and 0.5 hours by the physician, and the PRA per slot was 1.5 hours by the genetic counselor and 0.7 hours by the physician. Overall the physician spent 1.2 hours and the genetic counselor spent 2.3 hours per 1-hour patient slot. This is a total of 1.3 hours of professional face-to-face time and 2.2 hours of PRA or 3.5 hours overall for each 1-hour

\section{Genetic Counselor}

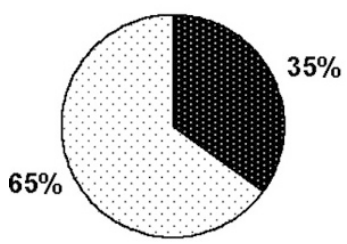

Di Pt Appt $\square$ PRA
Physician

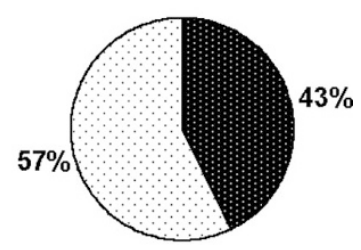

目 Pt Appt $\square$ PRA

Fig. 3. Clinical time breakdown.

patient slot (Fig. 3). Because of the batching of PRA tasks mentioned above, it is not possible to distinguish PRA time for new versus follow-up patients or to assign PRA time to any specific patient or group of patients. An earlier study in our institution, abandoned because recording of specific tasks by patient interfered severely with workflow, suggested that the time spent per patient was extremely variable even for patients with similar diagnoses.

\section{DISCUSSION}

This is the first real-time workflow study tracking not only clinical time (including preparation, face-to-face time and follow-up), but also research, teaching, and other activities for both genetic counselors and medical geneticists. Strengths of the current study include the prospective design, the excellent compliance with reporting, and the inclusion of genetic counselors. Weaknesses include a relatively short time period studied (10 weeks) and the limitation to a single institution with a relatively small number of providers.

We achieved our goal of real-time tracking of workflow in a genetics clinic including both physicians and genetic counselors, and providing more-detailed data than any previous study, but some limitations remained. No attempt was made to track time spent by secretarial and other support staff, some of whom are shared among different departments. Because time slots of $<15$ minutes proved impractical, and participants tended to batch certain tasks such as proofreading correspondence, PRA time cannot be matched to specific patients. Travel was not broken down according to the purpose (i.e., clinical outreach vs. research meetings) and in retrospect this data may have been useful.

Despite differences in methodology and recent changes in clinical practice, our results are strikingly similar to those of previous genetics workforce studies. Clinical genetics is still an extremely labor intensive discipline. The professionals involved have changed, but the total professional time spent is 7 hours per new patient and 3.5 hours per follow-up, which is very similar to the Bernhardt et al. ${ }^{1}$ study that reported 7 hours per new patient and 4 hours per follow-up. The face-to-face time is somewhat less, but the other PRA has increased, possibly reflecting the increasing paperwork involved with current 
medical practice. Although the Marshfield Clinic does have electronic medical records, the time savings are minimal because many of our patients still have outside records requiring HIPAA requests, review of scanned outside records is slower than reading paper records, dictation actually takes longer because lengthy outside records cannot be saved in the chart, but must be summarized for the electronic medical records, and proofreading time is extensive because we have a secretarial pool including individuals with little familiarity with genetic terminology. Furthermore, because many of our patients initially receive insurance denials for necessary services, professional time is required for insurance documentation and appeals. In our study, average face-to-face time with the clinical geneticist is only 63 minutes per new patient and 32 minutes per follow-up compared with 90 minutes per new patient and 79 minutes per follow-up in the Bernhardt et al. ${ }^{1}$ study and 75 minutes per new patient and 45 minutes per follow-up for general geneticists in the 2003 survey. ${ }^{2}$ The average combined face-to-face and PRA time spent by the physicians in our study was 2.4 hours per new patient, which is less than that in Pletcher et al. ${ }^{5}$ study. These trends may reflect efforts to streamline medical practice and increase use of non-physicians. The role of the genetic counselors was markedly increased in our study compared with the earlier 1987 study, ${ }^{1}$ probably encompassing much of the work done by residents, clinic coordinators, and social workers in the previous study. This is reflected not only by involvement of the counselor in $100 \%$ of patients in the current study compared with only $30 \%$ in the 1987 study, ${ }^{1}$ but also by increased face-to-face time with the genetic counselor ( $97 \mathrm{~min}$ per new patient and $49 \mathrm{~min}$ per follow-up in the current study vs. $61 \mathrm{~min}$ per new patient and 28 min per follow-up, counting only those who actually saw a genetic counselor in the Bernhardt study).

Although Bernhardt et al. ${ }^{1}$ recognized that nearly half of all clinical time was spent before and after the actual appointment, our study is the first to document such PRA separately for physicians and genetic counselors. PRA includes time spent in reviewing medical records of the patient and affected family members, literature review related to rare disorders, gathering of patient education materials and resource information for the patient, correspondence and telephone calls to insurance companies and referring physicians, as well as communication of lab results to the patient, additional counseling before or after the appointment, and reinforcing counseling provided via a detailed summary letter. The total PRA in our study was approximately 4.4 hours per new patient and 2.2 hours per follow-up, which is slightly more than in the Bernhardt et al. ${ }^{1}$ study. The 2003 genetics workforce survey did not specifically address PRA, but since physicians in general genetics indicated that they spent 28 hours per week in clinical activities, but the face-to-face patient time averaged to only 14.3 hours per week, it can be deduced that they spent approximately 14 hours per week on PRA. This can be compared with the current study in which (after correction for holidays and vacation) the physicians spent an average of 23 hours per week in clinical activities including 10 hours in face-to-face patient contact and 13 hours of PRA. For genetic counselors the time spent on patient care, particularly PRA, is even greater with a total of 32 hours per week devoted to clinical activities including 11 hours of faceto-face patient contact and 21 hours of PRA. This means that, on average, physicians spent 1.3 hours and genetic counselors spent nearly 2 hours on PRA for every hour of face-to-face patient time. Overall, only $38 \%$ of the clinical time is spent in direct patient contact and $62 \%$ is devoted to PRA. Clearly, the time required for PRA is a limiting factor for patient scheduling and reimbursement.

Like the 2003 genetics workforce survey, the current study also documented time devoted to other activities such as research and teaching. Although the current study involved a large multispecialty practice while most responders to the 2003 survey worked in an academic research center, the time distribution was remarkably similar. The average total work week for physicians in the current study was 54.1 hour that is comparable with the 55 hour average work week for general clinical geneticists in the 2003 survey. ${ }^{6}$ One physician in our study devoted an average of 28.8 hours per week to clinical activities (comparable with the average of 28 hours for general clinical geneticists in the 2003 survey), whereas the other spent only 17.2 hours per week on clinical activities because of greater research and administrative commitments. Although the physicians in the department had a total of 0.5 FTE support for research time, they actually spent the equivalent of 0.8 FTE total for research, much of which was outside of regular clinic hours. Much of the physician work time outside of regular clinic hours was used to meet research goals. Overall the physicians spent $43 \%$ of their time on clinical work, $29 \%$ on research, and $12 \%$ on travel, which included both clinical (i.e., travel to outreach) and research or CME (i.e., travel to a meeting) but was not counted in the clinical or research totals. Thus, the actual time spent on both clinical activities and research is greater than the numbers presented here, but comparisons with previous studies remain valid since they also made no specific provision for travel time. The remaining time distribution included $7 \%$ devoted to administrative tasks and 5\% to teaching. The time spent on administration was $0.2 \mathrm{FTE}$ that should be compared with the 0.1 FTE budgeted for administrative activities. Although both physicians in the current study have clinical faculty appointments, neither has any dedicated teaching time. The time spent on administration and teaching in the current study fell well within the $0-15 \%$ range reported by $85 \%$ of respondents to the 2003 survey.

Previous data on participation of genetic counselors in research are limited. In a 2006 survey, ${ }^{7} 53 \%$ of genetic counselors reported some research duties as part of their current job, with most of these spending about $25 \%$ of their time on research. Since the survey included some genetic counselors working as research coordinators, it is not surprising that the genetic counselors in the current study spent less time (only $\sim 7 \%$ of their total) on research. Considering that there are three genetic counselors, this comprises 0.2 FTE in exact agreement with their funded research commitment. The genetic counsel- 
ors spend significant time on administrative activities that are not addressed in our department budget.

The time and labor intensive nature of clinical genetics services, the large percentage of non-face-to-face time devoted to each patient, and the extensive involvement of genetic counselors (who in many states are still unable to bill for their services) and the simultaneous face-to-face involvement of the physician and genetic counselor (who cannot bill independently for the same service) have major impact on reimbursement in genetics clinics. In our study, a total of 1139 hours were devoted to patient care in which only 167.5 hours (15\%) were face-to-face time with the physician. This means that of every hour spent in providing clinical genetics services, only 9 minutes is billable. If face-to-face time spent by the counselor without the physician present also became reimbursable, the total billable time would increase to 14 minutes of each hour devoted to clinical work. Obviously, this makes it difficult for a genetics clinic to be self-supporting. Bernhardt and Pyeritz ${ }^{4}$ expressed hope that the development of billing based on relative value units (RVUs) and enhanced current procedural terminology (CPT) coding would improve billing for cognitive services such as clinical genetics, but major issues especially the lack of RVU credit for PRA remain unaddressed.

Because more than $50 \%$ of the session is always devoted to counseling, services provided by the physicians are billed on time spent face-to-face according to evaluation and management CPT codes for consults, new visits (if self-referred), or established patients. This method, which is similar to that applied to other cognitive services, allows the physicians to be reimbursed for their face-to-face time and avoids many coding pitfalls such as the fact that the genetics physical examination requires more detail in some areas and less in others compared with the service-based coding standards. Unfortunately, especially for cancer and prenatal patients, the physician face-toface time may be brief, justifying only a level 1 or 2 consult, yet the PRA time spent by both the physician and the genetic counselor may be extensive. Some of the newer codes that have been recently approved or are under consideration, such as those for genetic counseling and for obtaining a detailed family history, may be helpful in this regard, but were not in use during the time of this study.

Strategies that work to improve reimbursement in other settings may not be applicable. Increasing the number of patients seen will increase the demands on staff time much faster than it increases income, since $85 \%$ of the time spent on each patient is not billable. The use of genetic counselors as physician extenders is also problematic since, unlike physician associates or nurse practitioners, genetic counselors often cannot bill for their services. This issue was noted by Bernhardt and Pyeritz ${ }^{4}$ and despite the subsequent development of board certification for genetic counselors, the situation has not improved. A new genetic counseling CPT code has recently been approved, but is not yet accepted by insurers in our area. Genetic counselors are working to achieve licensure that may increase the chance of insurance reimbursement for their services in the future, but this will help only with the slightly over one third of their pro- fessional time that is spent face-to-face with patients. From the geneticist's viewpoint, billing for additional services such as phone calls and lengthy records review would be ideal, but realistically such billing is unlikely to be approved because of the cost. Increasing the proportion of face-to-face time by requiring return visits in place of phone calls or correspondence might be difficult to justify. A recent study has shown that telephone reporting of genetic test results does not compromise patient knowledge or psychological outcomes, costs less than in-person reporting, and is preferred by some patients. ${ }^{8}$ In our clinic setting, the decision to report most cancer genetic test results over the phone rather than in person was made about a year before the current study. This change has resulted in shortened waiting time for new clinic visits and has been essentially neutral from a reimbursement viewpoint, because the time slots previously reserved for these face-to-face follow-up visits have been devoted to other types of patients. Furthermore, many patients, including those who travel from a distance, have difficulty scheduling time off from their jobs, or simply want their results immediately rather than waiting several weeks for a follow-up visit, greatly prefer telephone reporting of results.

Some disciplines, facing similar problems, have elected to devote intensive effort to documentation of the services they provide and have been successful in improving reimbursement. While this approach has been most effective for procedures such as cardiac catheterization, it may be applicable to some cognitive services. Pediatric nephrologists receive a capitated monthly reimbursement for patients with end stage renal disease which is supposed to cover not only face-to-face time, but other services such as preparation time, ordering and review of laboratory work, patient care conferences, telephone management, and home visits. Through a joint effort of the American Academy of Pediatrics, the American Society of Pediatric Nephrology, and the Renal Physicians Association, and working within the RVU framework, pediatric nephrologists were able to document that services required for children with end-stage renal disease are more labor intensive than similar services for adults and the reimbursement was changed to reflect age differences. ${ }^{9}$ The development of a new code for detailed family history is an example of the application of this method to clinical genetics.

Decreasing the amount of PRA required would help with the billing issues and increasing the availability of genetic services, but is likely to prove challenging. Comparison of the current study with previous genetic workforce studies ${ }^{1,2}$ shows that PRA has actually increased over the past 20 years, whereas physician face-to-face time has decreased. The increased PRA may be a result of attempts to increase physician efficiency by shortening face-to-face time and delegating tasks, as well as the general increase in documentation required for the practice of medicine in recent years. The PRA issue is not unique to genetics. An observational study of primary care outpatient providers ${ }^{10}$ during office hours showed that they spent $55 \%$ of their workday in the examination room face-to-face with patients, $37 \%$ on other activities related to specific outpatients, 
and the remainder on administration, academic activities (teaching, CME, and research), and hospital rounds. Thus PRA for all patients combined occupied one third of the primary care providers' clinical time, with the most frequent activities being charting, telephone calls, and reviewing laboratory and radiographic reports. A PRA study of geriatric care providers showed that on the average they devote $18 \%$ of their clinical time to PRA. ${ }^{11}$ Their PRA consisted of brief (usually $<8$ minute) telephone, electronic, or written communications that were necessary due to the large number of medical problems, multiple medications, and multiple healthcare providers required by most elderly patients. One of the primary themes for PRA in general seems to be co-ordination of care since most of the PRA activities of the primary care providers and geriatricians would fall in that category. Possible explanations for the longer PRA time in clinical genetics compared with other disciplines include the need for general rather than systemspecific records review, the need to review medical records not only for the patient but also for the extended family, the frequency and variety of individually rare disorders in a genetics practice (which means time is needed for literature review, gathering of patient education materials, and identification of resources for patients with rare disorders), and the role of the genetics professional in education of the patient and referring physician. Although our study did not include detailed analysis of the specific activities performed by the geneticists and genetic counselors during their PRA time, a major difference in the pattern of the genetics PRA compared with that of the primary care physicians and geriatricians was evident. The genetics PRA tended to occur in much larger blocks often including several consecutive 15 minute time slots. The individual providers involved indicate that these were devoted to dictation and review of lengthy letters and reports designed for education of patients and referring physicians, protracted telephone counseling sessions with patients, review of extensive patient and family records, and literature searches on behalf of individual patients with rare or undiagnosed disorders. A moredetailed workflow study directed only at PRA might identify areas in which time could be saved or activities that could be delegated, but the cognitive activities (such as pre- and postvisit counseling and detailed educational communications with patients and referring providers) that distinguish genetics PRA from many other disciplines will continue to require extended professional time. Overall, a significant reduction in PRA may require a change in approach to delivery of genetic services. Suggestions for how this change could be made without compromising patient care are beyond the scope of this article. It can be hoped however that the delineation of the problem will be a first step toward a solution.

\section{CONCLUSIONS}

Clinical genetics services are time and labor intensive, not only because of the cognitive nature of the face-to-face service provided but also because of the extensive PRAs before and after the visit. Using real-time electronic documentation we have obtained results similar to previous workflow studies in terms of total time per patient, although there is a trend for decreasing face-to-face time with the patient and increasing PRA before and after the visit. The total time spent on clinical activities is 7 hours per new patient and 3.5 hours per follow-up or prenatal patient. Only $38 \%$ of the total clinical time is spent in face-to-face patient care and $60 \%$ of that is performed by genetic counselors (who, even in a best case scenario, can bill only for services not overlapping with the physician face-to-face time), leaving only $15-25 \%$ of the work potentially reimbursable. Furthermore, clinical geneticists and genetic counselors have other duties including research, administration, and teaching that are also inadequately reimbursed. Genetic counselors tend to limit their nonclinical activities to the available time, working an average of 43.5 hours per week with most of their after hours time devoted to PRA whereas physicians perform much of their research and teaching postclinic hours, resulting in a 54-hour average work week. Further work is needed to determine how the balance of face-to-face patient time, PRA, and other activities can be improved without compromising the quality of patient care.

\section{References}

1. Bernhardt BA, Weiner J, Foster EC, Tumpson JE, Pyeritz RE. The economics of clinical genetics services. II. A time analysis of a medical genetics clinic. Am J Hum Genet 1987;41:559-565.

2. Cooksey JA, Forte G, Benkendorf J, Blitzer MG. The state of the medical geneticist workforce: findings of the 2003 survey of American Board of Medical Genetics certified geneticists. Genet Med 2005;7:439-443.

3. Acheson LS, Wiesner GL, Zyzanski SJ, Goodwin MA, Stange KC. Family historytaking in community family practice: implications for genetic screening. Genet Med 2000;2:180-185.

4. Bernhardt BA, Pyeritz RE. The economics of clinical genetics services. III. Cognitive genetics services are not self-supporting. Am J Hum Genet 1989;44:288-293.

5. Pletcher BA, Jewett EAB, Cull WL, et al. The practice of clinical genet: a survey of practitioners. Genet Med 2002;4:142-149.

6. Cooksey JA, Forte G, Flanagan PA, Benkendorf J, Blitzer MG. The medical genetics workforce: an analysis of clinical geneticist subgroups. Genet Med 2006; 8:603-614.

7. Clark HM, Gamm J, Huether CA, Buncher CR, Pfau RI, Warren NS. Genetic counselors and research: current practices and future directions. Am J Med Genet C Semin Med Genet 2006;142:276-283.

8. Jenkins J, Calzone KA. Dimond E, et al. Randomized comparison of phone versus in-person BRCA1/2 predisposition genetic test result disclosure counseling. Genet Med 2007;9:487-495.

9. Arnold W, Alexander S. Cost, work, reimbursement, and the pediatric nephrologist in the United States Medicare/End-stage Renal Disease Program. Pediatr Nephrol 1997;11:250-275.

10. Gottschalk A, Flocke SA. Time spent in face-to-face patient care and work outside the examination room. Ann Fam Med 2005;3:488-493.

11. Farber J, Siu A, Bloom P. How much time do physicians spend providing care outside of office visits? Ann Intern Med 2007;147:693-698. 